\title{
Usage of fiber Bragg grating sensors in low earth orbit environment
}

\author{
Sang-Oh Park*a, Jin-Bum Moon ${ }^{\mathrm{a}}$, Yoen-Gwan Lee ${ }^{\mathrm{a}}$, Chun-Gon Kim ${ }^{\mathrm{a}}$, Shantanu Bhowmik ${ }^{\mathrm{b}}$ \\ ${ }^{a}$ Dept. of Aerospace Engineering, KAIST, Daejeon 305-701, Korea; $\quad{ }^{b}$ Aerospace Materials, \\ Delft University of Technology, Kluyverweg 1, Nederlands
}

\begin{abstract}
It is widely known that materials exposed to the severe low earth orbit (LEO) environment undergo degradations. For the evaluation of fiber Bragg grating (FBG) sensors in the LEO environment, the reflective spectrum change and the Bragg wavelength shift of FBG sensor were measured during aging cycles simulating the LEO environment. The LEO environment was simulated by high vacuum $\left(\sim 10^{-5}\right.$ Torr), ultraviolet (UV) radiation $(<200 \mathrm{~nm}$ wavelength), temperature cycling $\left(-30^{\circ} \mathrm{C} \sim 100^{\circ} \mathrm{C}\right.$ ), and atomic oxygen atmosphere (AO flux of $9.12 \times 10^{14}$ atoms $/ \mathrm{cm}^{2} / \mathrm{s}$ and kinetic energy of $\sim 0.04$ $\mathrm{eV})$. FBG sensor arrays were embedded into the graphite/epoxy composite material. Through the aging cycles simulated for the LEO environment, the change in the reflective spectrums and the Bragg wavelengths from FBG sensors were investigated.
\end{abstract}

Keywords: composites, low earth orbit, fiber Bragg grating

\section{INTRODUCTION}

Composite materials have many advantages such as high specific strength, stiffness, corrosion resistance, excellent fatigue resistance, and low coefficient of thermal expansion. Therefore the interests in composites have been increasing, and composite structural elements are widely used in making various components for automotive, aerospace, marine, and civil structures [1]. Especially, spacecrafts and aerospace structures require light weight, guaranteed high performance and stability during the operation. So, composites can be the suitable candidates that applied to space structures and systems owing to their extraordinary optical, thermal, electrical and mechanical characteristics $[2,3]$. In spite of these positive aspects of using composite materials, they may have difficulty in maintaining their outstanding performance against the harsh space environment [2]. And the fracture mechanism of composites is very complex under impact and internal damages are not easily detected compared to homogeneous metal materials. Hence, the structural health monitoring technology is necessary to be integrated in the composite structures for the reliability of aerospace structures.

The low Earth orbit (LEO) where a number of spacecrafts, including Space Shuttle and International Space Station (ISS) operate is very hazardous to composite materials. The LEO environment constituents consisting of high vacuum, ultraviolet (UV) radiation, thermal cycles, atomic oxygen (AO), charged particles, electromagnetic radiation, micrometeoroids, and space debris, significantly degrade the material characteristics of polymer matrix composite materials. After exposed to the severe LEO environment, the composite materials used in space structural components will undergo fatigue cracking caused by thermal cycling, surface erosion by $\mathrm{AO}$ attack, structural modification and mass loss by outgassing, modification of material properties by UV radiation, delamination by collisions with micrometeoroids and man-made debris with high velocities. Thus, in designing the space structures using composite materials, possible deterioration of mechanical properties caused by long-term exposure to the LEO space environment must be carefully considered. Accordingly, reliable understanding of the LEO environment at effects on composite materials, as well as the investigation of its characteristics are crucial.

Recently, as the integrated sensing part of composite structures, fiber optic sensors (FOSs) have been widely used due to their convenience of embedment without causing much mechanical defects in structures. Especially, fiber Bragg grating sensor (FBG) has the most excellent ability in multiplexing among various FOSs. If the gratings are exposed to external perturbations, the reflective spectrum and the Bragg wavelength of FBG are changed. By measuring the spectral transform and the wavelength shift, we can calculate the physical properties such as strain, temperature and other factors.

*sangfive@kaist.ac.kr; phone 8242 869-3759; fax 8242 869-3710; kaist.ac.kr

Sensors and Smart Structures Technologies for Civil, Mechanical, and Aerospace Systems 2008, edited by Masayoshi Tomizuka, Proc. of SPIE Vol. 6932, 69321T, (2008)

0277-786X/08/\$18 - doi: $10.1117 / 12.776631$

Proc. of SPIE Vol. 6932 69321T-1 
In this study, the change in the reflective spectrum and the Bragg wavelength shift of FBG sensor were measured for the evaluation of FBG sensor in LEO environment during the aging cycles simulated the LEO environment. Normally it is impossible to measure the internal strain with conventional strain gages, but the embedded FBG sensors can do. FBG sensor arrays were embedded into the graphite/epoxy composite materials. Through the LEO aging cycles, the measured reflectivity spectrums and Bragg wavelengths from FBG sensors were investigated for the evaluation of exposed graphite/epoxy composites.

\section{EXPERIMENTAL SET-UP AND PROCEDURES}

\subsection{FBG sensors}

There are many ways for FBG sensors. Fabrication method using a phase mask [4] is currently the most prevalently employed technique because it has an advantage of fabrication simplicity and hence suitable for mass production. A $\mathrm{KrF}$ excimer laser (ASX-750, MPB Co., Canada) was used as the UV light source. The setup for the fabrication process of FBGs is shown in Fig. 1. Each FBG sensor was fabricated using a single mode fiber (SMF) with high photosensitivity through a high temperature hydrogen loading process and was made to the reflectivity of $90 \%(10 \mathrm{~dB})$ in gage length of $10 \mathrm{~mm}$ by a phase mask (IBSEN Co., Denmark).

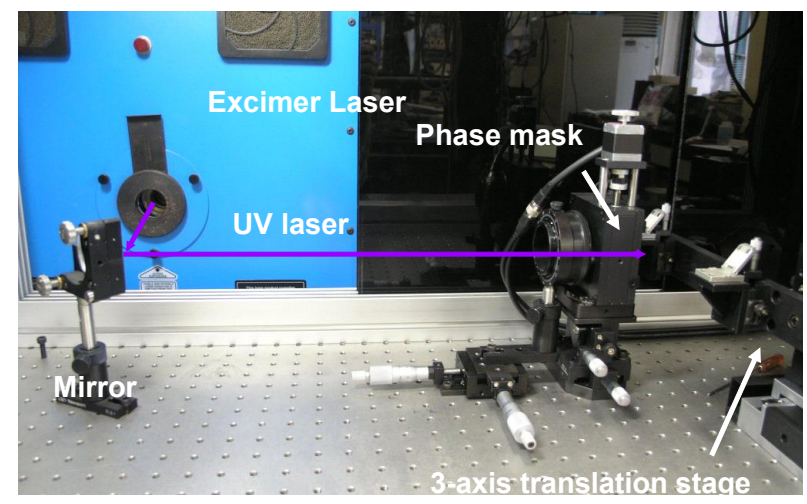

Fig. 1 Fabrication of FBG sensor.

A fiber Bragg grating is unique for the periodic changes of the refractive index property within the fiber gage length. The light of a specific Bragg wavelength, which is determined by the Bragg condition, is reflected at the Bragg grating part while lights with the other wavelengths pass through it. In Eq. (1), the Bragg wavelength is expressed as a multiple of the effective refractive index of the Bragg condition grating period [5]. Whenever grating is exposed to external perturbations, the Bragg period is either elongated or contracted, therefore resulting in the change of Bragg wavelength.

$$
\lambda_{B}=2 n_{e} \Lambda
$$

\subsection{Graphite/epoxy composite specimen}

Composite specimen was made of graphite/epoxy prepregs, CU-125NS (Hankook fiber glass co., Korea), using autoclave curing. The specimen has the lay-up configuration of $\left[0_{16}\right]$ with embedded seven FBG sensors. Each FBG was inserted in the interval of two prepreg sheets, $\left[0_{2} /\{0\} / 0_{2} /\{0\} / 0_{2} /\{0\} / 0_{2} /\{0\} / 0_{2} /\{0\} / 0_{2} /\{0\} / 0_{2} /\{0\} / 0_{2}\right]$ where " \{\} " means the direction of the embedded FBG. The $0^{\circ}$ angle refers to the horizontal direction (right and left) of the specimen, and the properties of laminates were given in Table 1. After curing, the average thickness measured at four edges was about $1.7 \mathrm{~mm}$. So, each FBG sensor was located with thickness of $0.2125 \mathrm{~mm}$ in the composite specimen. Fig. 2 shows the composite specimen with embedded FBG sensors.

Table 1 Stiffness properties of CU-125NS lamina

\begin{tabular}{|c|c|c|c|c|c|}
\hline $\mathrm{E}_{1}$ & $\mathrm{E}_{2}, \mathrm{E}_{3}$ & $\mathrm{G}_{12}, \mathrm{G}_{13}$ & $\mathrm{G}_{23}$ & $v_{1.2}, v_{13}$ & $v_{23}$ \\
\hline $135.4 \mathrm{GPa}$ & $9.6 \mathrm{GPa}$ & $4.8 \mathrm{GPa}$ & $3.2 \mathrm{GPa}$ & 0.31 & 0.52 \\
\hline
\end{tabular}




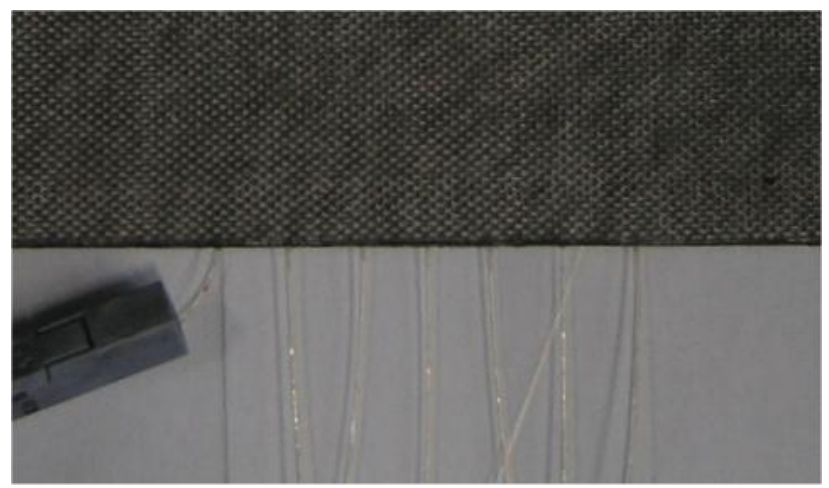

Fig. 2 Composite specimen with embedded FBG sensors.

\subsection{Low earth orbit environment}

The LEO environment included high vacuum, UV radiation, thermal cycling, and an AO. The vacuum chamber at the simulation facility was used to create atmospheric pressure on the order of $10^{-6}$ Torr near the LEO atmosphere. In Fig. 3, the schematic diagram of LEO space environment and the picture of simulation facility are shown.
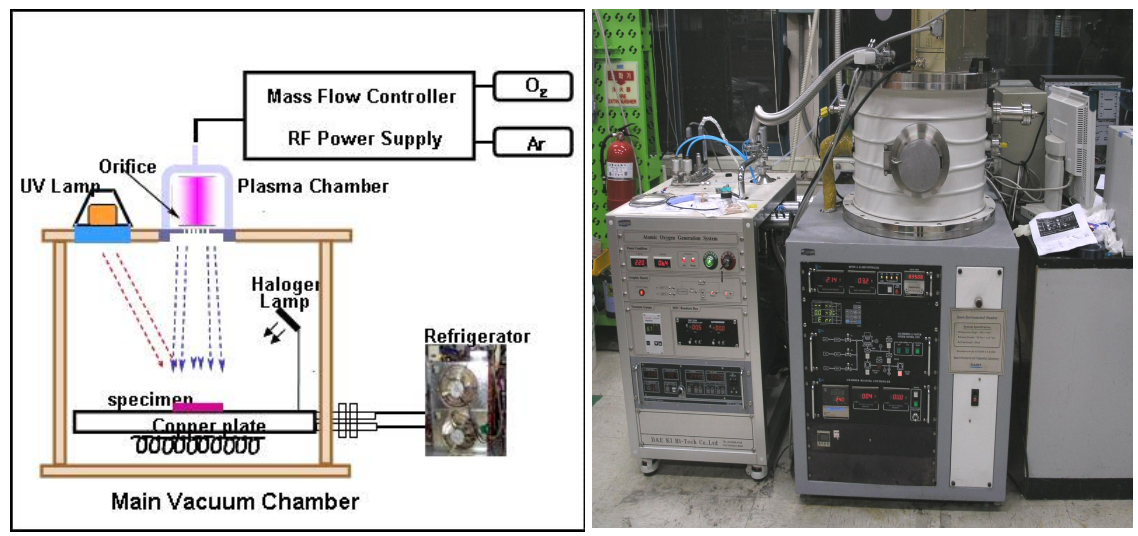

Fig. 3 LEO space environment simulation facility.

The primary electromagnetic radiation component of the LEO environment is UV radiation with wavelengths between 100 and $200 \mathrm{~nm}$ [6]. Exposure to UV radiation is likely to degrade thermo-optical and mechanical properties. At the simulation facility, the UV radiation of the LEO environment was simulated using a UV lamp that emitted radiation at a wavelength of close to $200 \mathrm{~nm}$ [7].

In the LEO environment, the thermal cycling between $+150^{\circ} \mathrm{C}$ and $-150{ }^{\circ} \mathrm{C}$ takes place on the surfaces of spacecraft as they face the sun or not. During the LEO environment simulation test, the sun-facing surface temperature was simulated by using a halogen lamp, set inside the chamber, while the shadow-facing surface temperature was simulated by using a refrigerator that circulated reusable coolant through a pipe in the chamber. The maximum and minimum operating temperatures for the thermal cycling simulation were $100{ }^{\circ} \mathrm{C}$ and $-30{ }^{\circ} \mathrm{C}$, respectively. With temperature increases occurring at about $22{ }^{\circ} \mathrm{C} / \mathrm{min}$ and temperature decreases occurring at about $5.3^{\circ} \mathrm{C} / \mathrm{min}$, a complete thermal cycle (from $30{ }^{\circ} \mathrm{C}$ to $100{ }^{\circ} \mathrm{C}$ and back to $-30^{\circ} \mathrm{C}$ ) took about 30 minutes.

At an altitude of about $300 \mathrm{~km}$, the density of $\mathrm{AO}$ ranges from approximately $8 \times 10^{9}$ atoms $/ \mathrm{cm}^{3}$ (during maximum solar activity) to approximately $2 \times 10^{9}$ atoms $/ \mathrm{cm}^{3}$ (during minimum solar activity). If a spacecraft orbits at that altitude at a 
velocity of $8 \mathrm{~km} / \mathrm{s}$, it would encounter AO particles with a kinetic energy of about $5 \mathrm{eV}$ at the nominal AO flux ranging approximately from $10^{14}$ to $10^{15}$ atoms $/ \mathrm{cm}^{2} \mathrm{~s}$ [2]. In the LEO simulation facility, a system was built for generating AO flux through a weakly ionized remote oxygen plasma with a radio-frequency (RF) plasma source. The plasma source was mainly operated through an $\mathrm{O}_{2}$ and $\mathrm{Ar}$ gas supply and a $600 \mathrm{~W}, 13.56 \mathrm{MHz} \mathrm{RF}$ power supply. When operating the AO generation system at an oxygen gas flow rate of $5.0 \mathrm{sccm}$ and an RF power of $200 \mathrm{~W}$, the maximum scanning of AO was found among other various gas-mixture flow rates. The maximum AO density was approximately $5.13 \times 10^{11}$ atoms $/ \mathrm{cm}^{3}$. The estimated temperature of the atomic oxygen neutrals in the oxygen plasma was approximately $0.04 \mathrm{eV}$. The calculated maximum AO flux was approximately $9.12 \times 10^{14}$ atoms $/ \mathrm{cm}^{2} / \mathrm{s}$ from calibration test using Kapton films in Eq. (2).

$$
\text { AO flux }\left(\text { atoms } / \mathrm{cm}^{2} \cdot \mathrm{s}\right)=\frac{\operatorname{Mass} \operatorname{Loss}(\mathrm{g})}{\operatorname{Density}\left(\mathrm{g} \cdot \mathrm{cm}^{3}\right) \times A O \text { reactivity coefficient }\left(\mathrm{cm}^{3} / \text { atom }\right) \times \operatorname{Area}\left(\mathrm{cm}^{2}\right) \times \operatorname{Time}(\mathrm{s})}
$$

\subsection{Experimental procedures}

The specimens were placed in the vacuum chamber and it took over 24 hours to keep high vacuum before exposed both to $\mathrm{AO}$ and to the simulated LEO environment. By the time the exposure began, the vacuum in the chamber had decreased to approximately $3.0 \times 10^{-5}$ Torr. During vacuuming the FBG interrogator (IS7000, Fibepro co., Korea) was used to measure the reflective spectrums and the Bragg wavelength shift according to applied environment. The reflective spectrums of FBG sensors were acquired at before and after vacuuming. The sampling frequency of Bragg wavelength shift was set up to $1 \mathrm{~Hz}$.

After vacuuming, during the 20 hours of AO exposure, specimens were simultaneously under high vacuum, UV radiation and thermal cycling. The AO influence of approximately $2.29 \times 10^{20}$ atoms $/ \mathrm{cm}^{2}$ took place. The simulated AO influence was very similar to $2.3 \times 10^{20}$ atoms $/ \mathrm{cm}^{2}$, the AO influence exposure of the STS-4 spacecraft [8]. Thermal cycling with UV radiation was simulated about 40 cycles by using the halogen lamp and the refrigerator. The thermal cycle process in vacuum chamber was controlled by LabVIEW (National Instrument ${ }^{\mathrm{TM}}$, U.S.A.) program and the maximum and minimum operating temperatures for the thermal cycling simulation were set up from $100{ }^{\circ} \mathrm{C}$ to $-30^{\circ} \mathrm{C}$, as shown in Fig. 4. During aging cycles simulated the LEO environment, the reflective spectrums and the Bragg wavelength shift were also measured using the FBG interrogator.

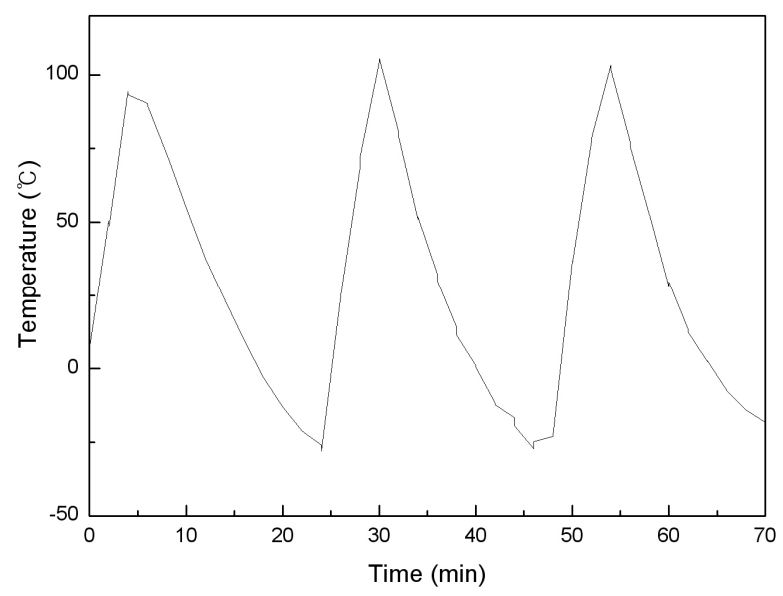

Fig. 4 Diagram of thermal cycling. 

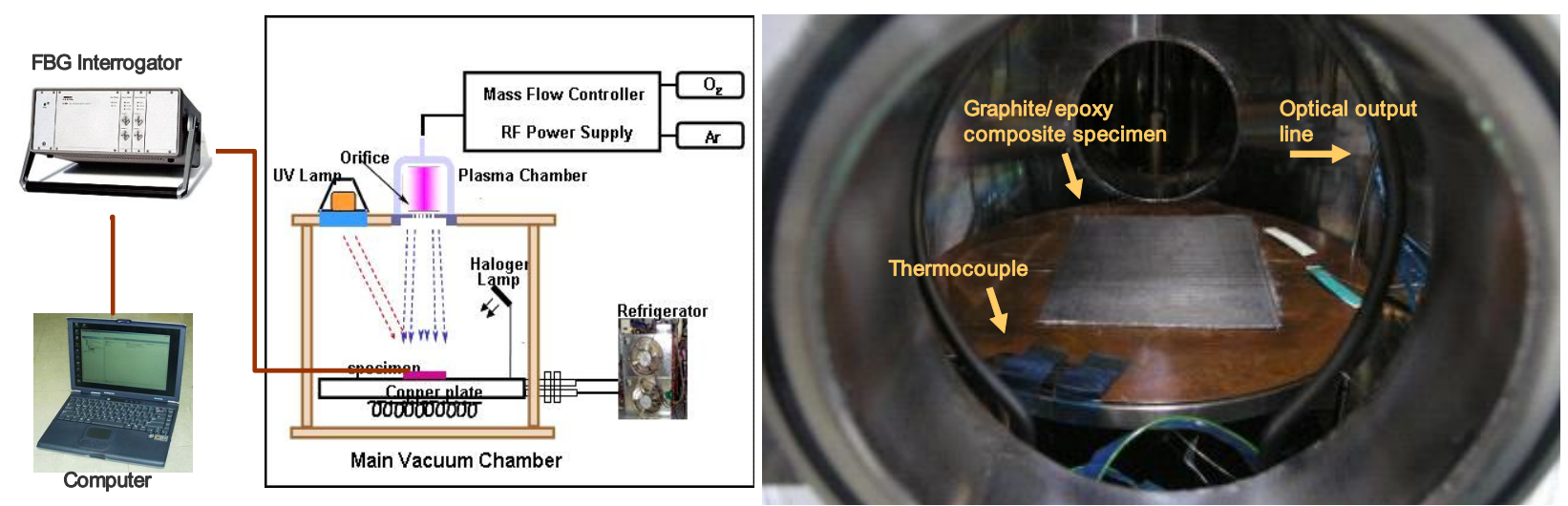

Fig. 5 Experimental set up for LEO test.

\section{DISCUSSION}

First, the single FBG sensor was tested in the LEO environment to investigate the characteristics of FBG sensor. Then two FBG sensor lines which were fabricated through an arc-fusion splicing were embedded into graphite/epoxy composites. The composites which was exposed to the LEO space environment undergo degradations. Unfortunately, the FBG embedded in mid plane of composite specimen was broken while setting the LEO experiment. So, the experimental signals of embedded six FBG sensors were acquired and were evaluated.

\subsection{Experimental results and observations of single FBG sensor}

Figure 6 shows the strain signal of the single FBG sensor during vacuuming. The strain measured by the FBG sensor extremely dropped down to $-229.68 \mu \varepsilon$ at the beginning of vacuuming and increased up to $10.04 \mu \varepsilon$. The sudden low pressure made the optical fiber shrink and the continued high vacuum induced the relaxation of the FBG sensor.

During the test, there was no distortion of the reflective spectrum and the signal measured by the FBG sensor showed a good relation with the thermal cycling. But the reflective spectrum was slowly changed. The reflective spectral intensity increased during vacuuming, and decreased during the aging cycles simulated the LEO environment, as shown in Fig. 7. The high vacuum made the optical fiber relax. So, the diameter as well as the length of optical fiber line was extended a little and the ray could pass more easily in the optical line. On the other hand, the LEO aging cycles weakened the intensity of the Bragg grating and the reflective spectrum of FBG degraded a little. Therefore, it was conformed that it could be possible to measure the internal strain by FBG sensor in the LEO space environment.

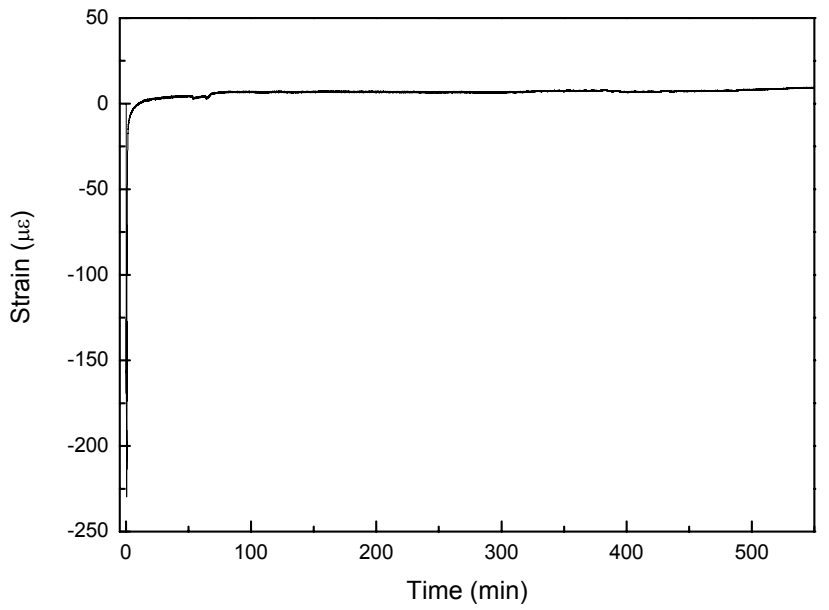

Fig. 6 Strain signal of single FBG sensor during vacuuming.

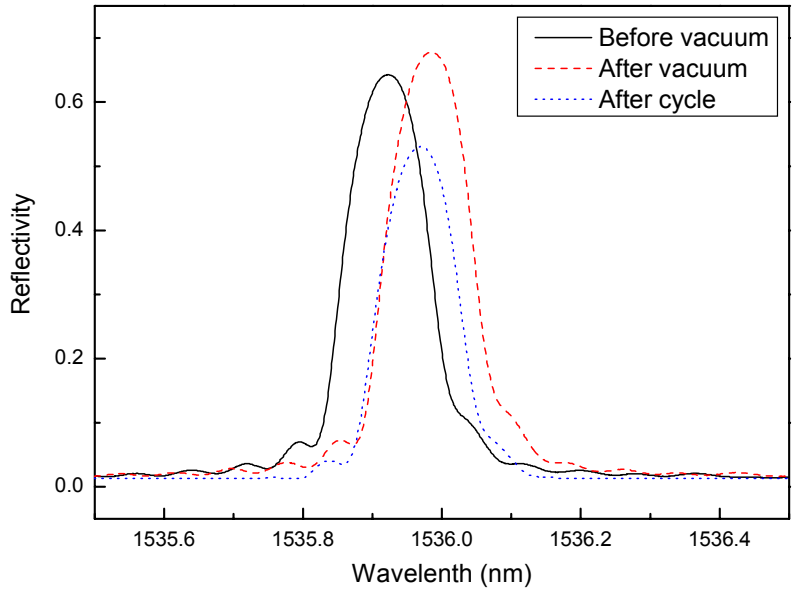

Fig. 7 Reflective spectrum change of single FBG sensor. 


\subsection{Experimental results and observations of graphite/epoxy composites with embedded FBG sensors}

The strain measured by the embedded FBG sensors in graphite/epoxy composites also dropped similarly to that of the single FBG sensor on the beginning of vacuuming, but strain signals were fluctuated differently to that of the single FBG sensor as time went on, as shown Fig. 8. It means that the high vacuum induced the outgassing of graphite/epoxy composite specimen and the outgassing deformed the shape of composite specimen, which caused by the failure of dimensional stability.

Figure 9 shows the wavelength changes of the embedded FBG sensors during LEO aging cycles. As the aging cycle increased, the range of the wavelength change decreased. Because the epoxy matrix and the fibers in composite materials have different CTEs, this extreme thermal cycling caused microcracking and other degradation of material properties. Figure 10 shows the wavelength change of the embedded FBG sensors at rising and falling of the 2nd and the 40th cycle. As the aging cycle increased, the FBG sensor which was located at the upper position of the composite specimen responded more insensibility to the aging cycles. Especially, when AO collides with polymeric materials, the degradation of mechanical, thermal, and optical properties occurred from the upper surface of composite specimen.

The reflective spectrums of embedded FBG sensors were also slowly changed. The most reflective spectral intensity increased during vacuuming. However, the reflective spectral intensities of the three embedded FBG sensors which were located at the upper position of the composite specimen decreased during the aging cycles of the LEO environment, as shown in Fig. 11. It means that the thickness penetrated by AO in the composite specimen could be measured.

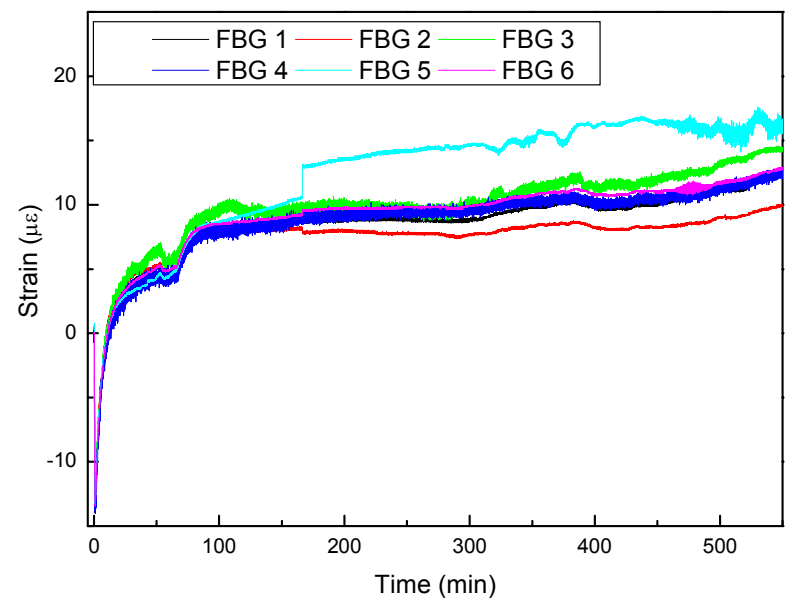

Fig. 8 Strain signals of embedded FBG sensors during vacuuming.

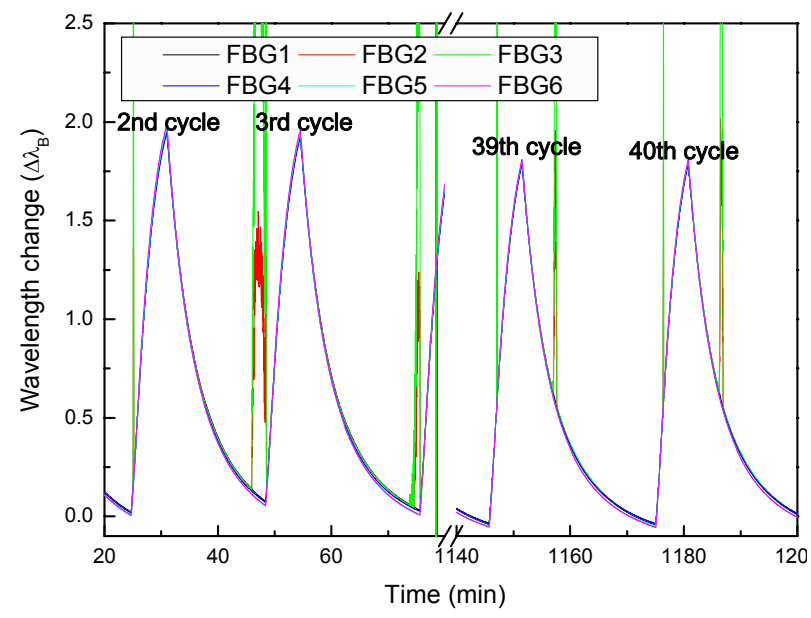

Fig. 9 Wavelength changes of embedded FBG sensors during LEO aging cycles. 

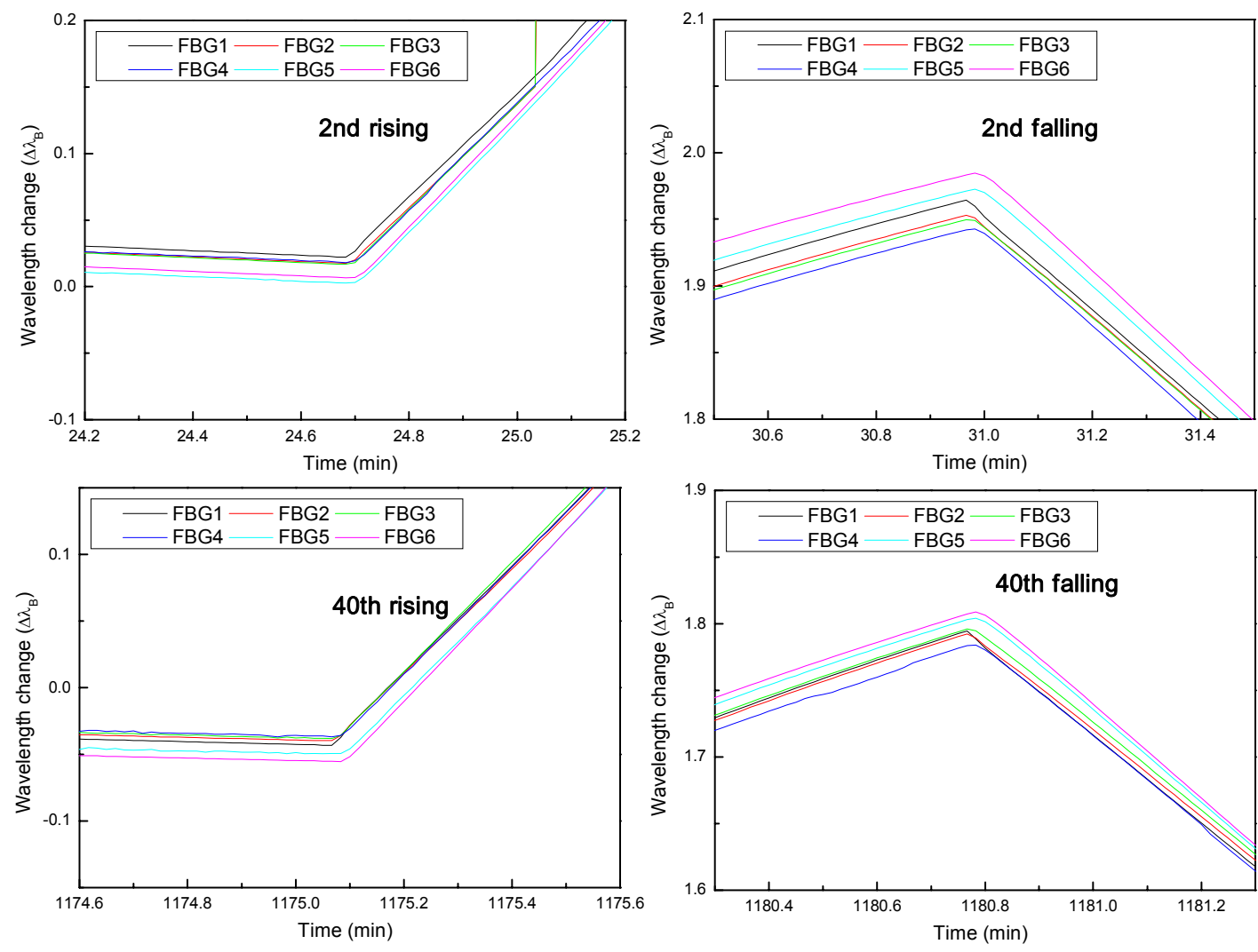

Fig. 10 Wavelength changes of embedded FBG sensors at rising and falling of 2nd and 40th cycle.
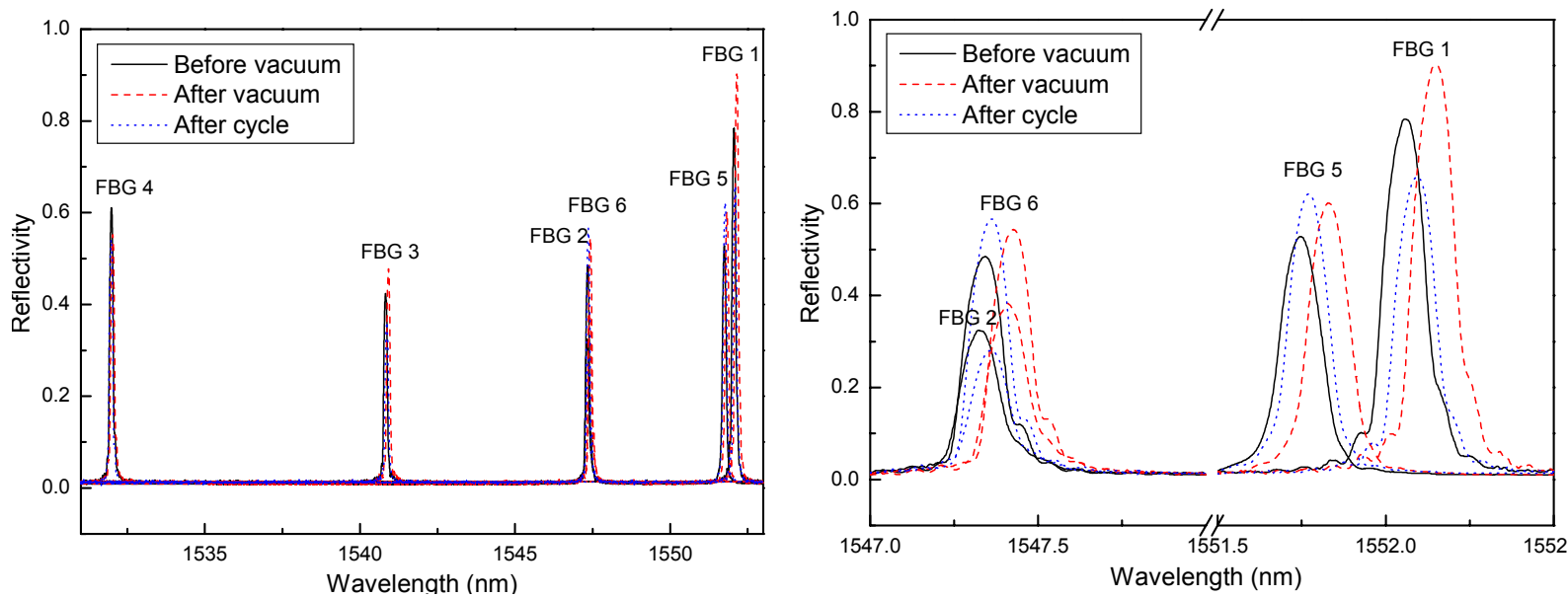

Fig. 11 Reflective spectrum changes of embedded FBG sensors. 


\section{CONCLUSIONS}

In this paper, the reflective spectrum changes and the Bragg wavelength shifts of FBG sensor were measured during vacuuming and LEO aging cycles for the evaluation of FBG sensors in LEO environment. Therefore, it was conformed that it could be possible to measure the internal strain by FBG sensor in the LEO space environment.

Then, FBG sensors were embedded into the graphite/epoxy composite specimen. The fluctuation of strain signals showed that the high vacuum induced the outgassing of graphite/epoxy composite specimen and deformed the shape of composite specimen. Through aging cycles simulating the LEO environment, the decreased range of wavelength change meant the degradation of material properties of graphite/epoxy composites. And the reflective spectrum changes made it possible to measure the thickness penetrated by AO.

From the results, it was demonstrated that FBG sensors could be successfully adopted to the aerospace structures for their structural health monitoring by embedding.

\section{ACKNOWLEDGMENTS}

This work was supported by the Korea Science and Engineering Foundation (KOSEF) grant funded by the Korea government (MOST) (No. 2007-04453).

\section{REFERENCES}

[1] R. F. Gibson, [Principles of Composite Material Mechanics], McBraw-Hill, Inc., 13 (1994).

[2] J. I. Kleiman, Z. A. Iskanderova, F. J. Perez, and R. C. Tennyson, "Protective Coatings for LEO Environment in Spacecraft Applications, " Surface and Coatings Technology, 76-77, 827-834 (1995).

[3] E. Grossman, and I. Gouzman, "Space Environment Effects on Polymers in Low Earth Orbit, " Nuclear Instruments and Methods in Physics Research B, 208, 48-57 (2003).

[4] K. O. Hill, B. Malo and F. Bilodeau, et al., "Bragg gratings fabricated in monomode photosensitive optical fiber by UV exposure through a phase mask," Applied Physics Letters, 62(10), 1035-1037 (1993).

[5] E. Udd, [Fiber Optic Smart Structures], John Wiley and Sons, (1995).

[6] R. C. Tennyson, "Composites in Space - Challenges and Opportunities," Proceedings of ICCM-10, (1995).

[7] J. H. Han and C. J. Kim, "Low Earth Orbit Space Environment Simulation and Its Effects on Graphite/Epoxy Composites, " Composite Structures, 72(2), 218-226 (2006).

[8] J. C. Gregory, and P. N. Peters, "The Reactions of $5 \mathrm{eV}$ Oxygen Atoms with Polymeric and Carbon Surfaces in Orbit, " Polymer Preprints, 28(2), 459-460 (1987). 